\title{
SLICE STARLIKE FUNCTIONS OVER QUATERNIONS
}

\author{
ZHENGHUA XU AND GUANGBIN REN
}

\begin{abstract}
In this paper, we initiate the study of the geometric function theory for slice starlike functions over quaternions and its subclasses. This allows us to answer negatively some questions about the Bieberbach conjecture, the growth, distortion, and covering theorems for slice regular functions. Precisely, we find that the Bieberbach conjecture holds true for slice starlike functions in contrast to the fact that the Bieberbach conjecture fails for biholomorphic starlike mappings in higher dimensions. We also establish some sharp versions of the growth, distortion, and covering theorems for quaternions.
\end{abstract}

\section{INTRODUCTION}

As a generalization of one complex variable, the theory of slice regular functions over quaternions is initiated by Gentili and Struppa [18, 19] and further developed in [7, 20, 23.

Many results of the geometric function theory of one complex variable can be extended to the setting of slice regular functions over quaternions such as Schwarz-Pick lemma [5], Bohr theorem [12], BlochLandau theorem 11, 48, Landau-Toeplitz theorem [21, Borel-Carathéodory theorem [39, Julia theory 40. and invariant metrics for the quaternionic Hardy space 3. However, some new phenomena occur in the quaternionic setting. For example, it was proven that no Riemannian metric is invariant under all slice regular self-maps of the quaternionic ball [3].

However, some sharp results are unfortunately restricted to the subclass of slice regular functions preserving one slice and some conjectures are believed to hold true only in this function class. For examples, Gal, González-Cervantes, and Sabadini in [15] suspect that the condition of preserving one slice is necessary for the Bieberbach conjecture. In 41, Ren and Wang establish the growth, distortion, and covering theorem for slice regular functions that preserve one slice via a so-called convex combination identity and raised the open question whether the condition of preserving one slice is also necessary.

In contrast to the wealthy results of geometric function theory for holomorphic starlike functions, there is essentially no result in the setting of quaternions except for [27]. One reason is that without the assumption preserving one slice, all tools such as the splitting lemma and the convex combination identity fail. The other reason is that the study of the slice starlike functions depends heavily on a deep close relation between the regular multiplication and classical point-wise multiplication, which is unfortunately unclear up to now.

2010 Mathematics Subject Classification. Primary 30G35; Secondary 30C50.

Key words and phrases. starlike function, quaternions, Bieberbach conjecture, Fekete-Szegö inequality, growth and distortion theorems, Bloch-Landau theorem.

This work was supported by the NNSF of China (11371337). 
The main aim of this article is to study the geometric function theory for slice starlike functions over quaternions. In particular, we shall establish some coefficient estimates and the theorems concerning growth, distortion, and covering for slice starlike functions over quaternions.

One of the most known coefficient estimates for univalent functions is the celebrated Bieberbach conjecture proved by de Branges [9] in 1985.

Theorem A. Let $f(z)=z+\Sigma_{n=2}^{+\infty} a_{n} z^{n}$ be an injective holomorphic function on the open unit disk $\mathbb{D}=\{z \in \mathbb{C}:|z|<1\}$, then

$$
\left|a_{n}\right| \leq n, \quad n=2,3, \ldots
$$

with strict inequality for all $n$ unless $f$ is a rotation of the Koebe function.

Cartan [6] pointed out that this result fails in several complex variables for biholomorphic functions as shown by providing a counterexample (cf. 24). It is quite nature to pose the Bieberbach conjecture in higher dimensions as follows:

Let $\Omega$ be the open unit ball or polydisk in $\mathbb{C}^{n}(n \geq 2)$ and let $f: \Omega \rightarrow \mathbb{C}^{n}$ be a biholomorphic starlike mapping normalized by $f(0)=0$ and $D f(0)=I d$. Then

$$
\frac{\left\|D^{m} f(0)\left(z^{m}\right)\right\|}{m !} \leq m, \quad m=2,3, \ldots,\|z\|=1,
$$

where $\|\cdot\|$ is the norm of the unit ball or polydisk.

Unfortunately, this conjecture also fails for the unit ball. For example, take

$$
f(z)=\left(z_{1}+a z_{2}^{2}, z_{2}\right), \quad z=\left(z_{1}, z_{2}\right) \in B^{2},
$$

where $B^{2}=\left\{z \in \mathbb{C}^{2}:\|z\|=\sqrt{\left|z_{1}\right|^{2}+\left|z_{2}\right|^{2}}<1\right\}$. If $a=3 \sqrt{3} / 2$, then $f\left(B^{2}\right)$ is starlike (see [45] Example 5]). However, for $z=(0,1)$, we have $\left\|D^{2} f(0)\left(z^{2}\right)\right\| / 2=a>2$.

Recently, a much weak positive result for the Bieberbach conjecture has been given for a subclass of starlike mappings on the unit ball [36]. So far there still lacks sharp results in higher dimensions.

Our first main result is to establish a sharp version of the de Branges theorem for quaternions. In contrast to the case of several complex variables, it turns out that the Bieberbach conjecture holds true for slice starlike functions. This seems to be the first sharp de Branges theorem in higher dimensions.

Now we state the definition of slice starlike functions. Let $f$ be a slice regular function in the unit ball $\mathbb{B}$ of quaternions $\mathbb{H}$, normalized by $f(0)=0$ and $f^{\prime}(0)=1$. We further assume that $\mathcal{Z}_{f}=\{0\}$, where $\mathcal{Z}_{f}$ denotes the zero set of $f$. Notice that this condition is weaker than $f$ is injective. Denote this function class by $\mathcal{S}$, i.e.,

$$
\mathcal{S}=\left\{f: \mathbb{B} \rightarrow \mathbb{H}: f \text { is slice regular such that } \mathcal{Z}_{f}=\{0\} \text { and } f^{\prime}(0)=1\right\} .
$$

The set of slice starlike functions is defined as

$$
\mathcal{S}^{*}=\left\{f: \mathbb{B} \rightarrow \mathbb{H}: f \in \mathcal{S} \text { such that } \operatorname{Re}\left(f(q)^{-1} q f^{\prime}(q)\right)>0 \text { on } \mathbb{B}\right\} .
$$

In one complex variable case, a holomorphic function $f$ in $\mathcal{S}^{*}$ is exactly the starlike function (see Theorem 3.2). Moreover, it is proven that the slice regular extension of any holomorphic starlike function belongs to the function class $\mathcal{S}^{*}$ (see Example 3.4). Hence, the results obtained for $\mathcal{S}^{*}$ in this paper extend corresponding results for holomorphic starlike functions to high dimensions. 
In fact, the algebraic condition in $\mathcal{S}^{*}$ can also be described as a geometric restriction that the element in $\mathcal{S}^{*}$ is a slice regular function for which its modulus is strictly increasing in the radial. Besides, we give some equivalent descriptions of the slice starlike function of order $\alpha$ (see Lemma 3.7) which allow us to present a new characterization of holomorphic starlike functions in one complex variable case (see Corollary [3.8) and find that the so-called slice starlike in [16. Definition 3.17] and algebraically starlike in [27, Definition 5.20] are equivalent.

Let us introduce two important subclasses of slice regular functions. Denote by $\mathbb{S}$ the unit 2-sphere of purely imaginary quaternions. For every $I \in \mathbb{S}$, denote by $\mathbb{C}_{I}$ the complex plane $\mathbb{R} \oplus I \mathbb{R}$, isomorphic to $\mathbb{C}$. Denote by $\mathbb{B}_{I}$ the intersection $\mathbb{B} \cap \mathbb{C}_{I}$. One subclass of slice regular functions is given by

$$
\mathcal{N}(\mathbb{B})=\left\{f: \mathbb{B} \rightarrow \mathbb{H}: f \text { is slice regular such that } f\left(\mathbb{B}_{I}\right) \subset \mathbb{C}_{I} \text { for all } I \in \mathbb{S}\right\}
$$

Another subclass is given by

$$
\mathcal{V}(\mathbb{B})=\left\{f: \mathbb{B} \rightarrow \mathbb{H}: f \text { is slice regular such that } f\left(\mathbb{B}_{I}\right) \subset \mathbb{C}_{I} \text { for some } I \in \mathbb{S}\right\}
$$

For the normalized and injective function $f \in \mathcal{V}(\mathbb{B})$, a quaternionic version of de Branges theorem was established and a natural question was raised if $\mathcal{V}(\mathbb{B})$ is the largest class of injective slice regular functions in which the Bieberbach conjecture holds [15].

In the present paper, we shall give a negative answer about Bieberbach conjecture in quaternions raised by Gal et al. in [15, p. 1359] and show that the Bieberbach conjecture holds for slice starlike functions as follows.

Theorem 1.1. Let $f(q)=q+\sum_{n=2}^{+\infty} q^{n} a_{n} \in \mathcal{S}^{*}$. Then

$$
\left|a_{n}\right| \leq n, \quad \text { for all } n=2,3, \ldots
$$

Equality $\left|a_{n}\right|=n$ for a given $n \geq 2$ holds if and only if

$$
f(q)=q(1-q u)^{-* 2}, \quad \forall q \in \mathbb{B},
$$

for some $u \in \partial \mathbb{B}$.

Note that, by Theorem 1.1 and Example 3.1 one can find that the Bieberbach conjecture holds for many injective slice regular functions which are not in $\mathcal{V}(\mathbb{B})$. Moreover, the Bieberbach conjecture over quaternions still holds for slice close-to-convex functions (see Theorem 4.3).

Related to the Bieberbach conjecture, Fekete and Szegö in 14 proved a striking inequality

$$
\left|a_{3}-\lambda a_{2}^{2}\right| \leq \begin{cases}3-4 \lambda, & \text { if } \lambda \in(-\infty, 0), \\ 1+2 e^{-2 \lambda /(1-\lambda)}, & \text { if } \lambda \in[0,1), \\ 4 \lambda-3, & \text { if } \lambda \in[1,+\infty),\end{cases}
$$

for any injective holomorphic function $F(z)=z+\Sigma_{n=2}^{+\infty} a_{n} z^{n}$ on $\mathbb{D}$. Moreover, inequality (1.1) is sharp for each $\lambda \in \mathbb{R}$. For related complex versions of Theorem 1.2 we refer the reader to the literature 33, 35] and [13].

In this paper, we shall establish the Fekete-Szegö type inequality for slice starlike functions as follows. 
Theorem 1.2. Let $f(q)=q+\sum_{n=2}^{+\infty} q^{n} a_{n} \in \mathcal{S}^{*}$. Then, for any $\lambda \in \mathbb{H}$,

$$
\left|a_{3}-\lambda a_{2}^{2}\right| \leq \max \{1,|4 \lambda-3|\}
$$

This estimate is sharp for each $\lambda$. Equality occurs if

$$
f(q)=q(1-q u)^{-* 2}, \quad q \in \mathbb{B},
$$

for any $u \in \partial \mathbb{B}$.

Note that if $\lambda=0$ in Theorem 1.2, then this result is already obtained in Theorem 1.1

As a very important consequence of Theorem $\mathrm{A}$ for the second-order coefficient, one may deduce the following well-known growth and distortion theorems.

Theorem B. Let $F(z)=z+\Sigma_{n=2}^{+\infty} a_{n} z^{n}$ be an injective holomorphic function on $\mathbb{D}$. Then for each $z \in \mathbb{D}$, the following inequalities hold:

$$
\begin{aligned}
& \frac{|z|}{(1+|z|)^{2}} \leq|F(z)| \leq \frac{|z|}{(1-|z|)^{2}} \\
& \frac{1-|z|}{(1+|z|)^{3}} \leq\left|F^{\prime}(z)\right| \leq \frac{1+|z|}{(1-|z|)^{3}} \\
& \frac{1-|z|}{1+|z|} \leq\left|\frac{z F^{\prime}(z)}{F(z)}\right| \leq \frac{1+|z|}{1-|z|}
\end{aligned}
$$

Moreover, equality holds for one of these six inequalities at some $z_{0} \in \mathbb{D} \backslash\{0\}$ if and only if

$$
F(z)=\frac{z}{\left(1-e^{i \theta} z\right)^{2}}, \quad \forall z \in \mathbb{D},
$$

for some $\theta \in \mathbb{R}$.

However, the growth and distortion theorems fail generally in $\mathbb{C}^{n}(n \geq 2)$ and only hold for some subclass such as starlike or convex mappings (cf. [25, 28]). Based on Theorem B] the growth and distortion theorems were formulated for normalized injective slice regular functions in the special class $\mathcal{N}(\mathbb{B})$ (see [15, Theorem 3.11]). See 41] for further extensions to normalized injective (on $\mathbb{B}_{I}$ ) slice regular functions in $\mathcal{V}(\mathbb{B})$.

In this paper, we shall answer an open problem in 41] and show that the class $\mathcal{V}(\mathbb{B})$ is not the largest subclass of slice regular functions such that the corresponding growth, distortion, and covering theorems hold. Indeed, it is proven that the growth, distortion, and covering theorems are valid in a tighter form for $\mathcal{S}^{*}$ by applying a new growth theorem for the Carathéodory class in the quaternionic setting. They are essentially the first significant theorems belonging to the theory of quaternions itself for that the Taylor coefficients of slice regular functions need not belong to one slice.

Notice that the close analytic relation between convex and starlike functions known as Alexander's theorem asserts that $F$ is convex if and only if $z F^{\prime}(z)$ is starlike for the holomorphic function $F$ on $\mathbb{D}$ normalized by $F(0)=0$ and $F^{\prime}(0)=1$. In view of this result, we give another coefficient estimate for a subclass of slice regular functions from Theorem 1.1. 
Theorem 1.3. Let $f(q)=q+\sum_{n=2}^{+\infty} q^{n} a_{n}$ be a slice regular function on $\mathbb{B}$ such that $q f^{\prime}(q) \in \mathcal{S}^{*}$. Then

$$
\left|a_{n}\right| \leq 1, \text { for all } n=2,3, \ldots
$$

Equality $\left|a_{n}\right|=1$ for a given $n \geq 2$ holds if and only if

$$
f(q)=q(1-q u)^{-*}, \quad \forall q \in \mathbb{B},
$$

for some $u \in \partial \mathbb{B}$.

Theorem 1.3 implies directly the following growth theorem:

$$
|f(q)| \leq \frac{|q|}{1-|q|}, \quad \forall q \in \mathbb{B},
$$

for any slice regular function $f$ on $\mathbb{B}$ such that $f(0)=0$ and $q f^{\prime}(q) \in \mathcal{S}^{*}$.

For normalized convex functions $F$ on $\mathbb{D}$, there holds a sharper growth theorem

$$
\frac{|z|}{1+|z|} \leq|F(z)| \leq \frac{|z|}{1-|z|}, \quad \forall z \in \mathbb{D}
$$

which was generalized to $\mathbb{C}^{n}$ (see e.g., 28, 25, 37]).

Usually, inequality (1.5) is just a consequence of the following distortion theorem in one and several complex variables.

Theorem 1.4. Let $f$ be a slice regular function on $\mathbb{B}$ such that $q f^{\prime}(q) \in \mathcal{S}^{*}$. Then

$$
\frac{1}{(1+|q|)^{2}} \leq\left|f^{\prime}(q)\right| \leq \frac{1}{(1-|q|)^{2}}, \quad \forall q \in \mathbb{B} .
$$

Estimates are sharp. For each $q \in \mathbb{B} \backslash\{0\}$, equality occurs if

$$
f(q)=q(1-q u)^{-*}, \quad q \in \mathbb{B},
$$

for some $u \in \partial \mathbb{B}$.

However, the method adopted in one and several complex variables cannot be used to our setting due to higher dimensions as well as the non-commutativity of quaternions and we do not know if the image of $q f^{\prime}(q)$ is convex for any $f \in \mathcal{S}^{*}$. Fortunately, we can obtain a Koebe type theorem for slice regular functions with convex image as follows.

Theorem 1.5. Let $f$ be a slice regular function on $\mathbb{B}$ with convex image and $f^{\prime}(0)=1$. Then $f(\mathbb{B})$ contains an open ball centered at $f(0)$ of radius $1 / 2$. Moreover, the constant $1 / 2$ is optimal.

It is worth mentioning that many methods in complex cases may fail in the quaternionic setting since the regularity does not keep under usual product and composition of two slice regular functions due to the non-commutativity of quaternions. For example, the subordination argument in 29 is not suitable to prove Theorem 5.4 In addition, the non-commutativity of quaternions forces us to formulate new auxiliary functions as in the proof of Theorem 3.11 and to explore new methods as in Theorems 5.4 and 1.5 Especially, Proposition 3.6 sets up a bridge of point-wise and regular multiplication for slice regular functions.

The remaining part of this paper is organized as follows. 
In Sect. 2, we set up basic notation and give some preliminary results from the theory of slice regular functions over quaternions. At the end of this section, we introduce the concepts of slice starlike functions of order $\alpha$ and slice close-to-convex functions.

In Sect. 3, we first offer some examples in the space $\mathcal{S}^{*}$ and establish some useful lemmas to prove Theorems 1.1 and 1.2. We also recall a Schwarz lemma from [19] and establish the Rogosinski lemma for slice regular functions which is a sharpened form of the Schwarz lemma.

In Sect. 4, we give a coefficient estimate for the Carathéodory class in the quaternionic setting and prove Theorems 1.1 and 1.2

In Sect. 5, we shall establish the growth and distortion theorems for some classes of slice regular functions in the quaternionic setting corresponding to Theorem B. Moreover, we formulate a result of Hayman (see Theorem 5.7) which is a precise version of the growth theorem.

In Sect. 6, we shall give some results involving the radius problems for slice regular functions. Specially, we establish the quaternionic analogs of Bohr theorem and Rogosinski theorem which are of independent interest. As an application of the growth theorem, the Koebe type one-quarter theorem for the class $\mathcal{S}^{*}$ is also obtained. Finally, we prove Theorem 1.5 by Proposition 3.6 which allows to establish a Bloch-Landau theorem for slice regular functions with convex image.

\section{Preliminaries}

In this section, we recall some necessary definitions and preliminary results on slice regular functions. To have a more complete insight on the theory, we refer the reader to the monograph [8, 22].

Let $\mathbb{H}$ denote the non-commutative, associative, real algebra of quaternions with standard basis $\{1, i, j, k\}$, subject to the multiplication rules

$$
i^{2}=j^{2}=k^{2}=i j k=-1 .
$$

Every element $q=x_{0}+x_{1} i+x_{2} j+x_{3} k$ in $\mathbb{H}$ is composed by the real part $\operatorname{Re}(q)=x_{0}$ and the imaginary part $\operatorname{Im}(q)=x_{1} i+x_{2} j+x_{3} k$. The conjugate of $q \in \mathbb{H}$ is then $\bar{q}=\operatorname{Re}(q)-\operatorname{Im}(q)$ and its modulus is defined by $|q|^{2}=q \bar{q}=|\operatorname{Re}(q)|^{2}+|\operatorname{Im}(q)|^{2}$. We can therefore calculate the multiplicative inverse of each $q \neq 0$ as $q^{-1}=|q|^{-2} \bar{q}$. Every $q \in \mathbb{H}$ can be expressed as $q=x+y I$, where $x, y \in \mathbb{R}$ and

$$
I=\frac{\operatorname{Im}(q)}{|\operatorname{Im}(q)|}
$$

if $\operatorname{Im} q \neq 0$, otherwise we take $I$ arbitrarily such that $I^{2}=-1$, where $I$ is an element of the unit 2-sphere of purely imaginary quaternions

$$
\mathbb{S}=\left\{q \in \mathbb{H}: q^{2}=-1\right\}
$$

For every $I \in \mathbb{S}$, we will denote by $\mathbb{C}_{I}$ the plane $\mathbb{R} \oplus I \mathbb{R}$, isomorphic to $\mathbb{C}$, and, if $\Omega \subset \mathbb{H}$, by $\Omega_{I}$ the intersection $\Omega \cap \mathbb{C}_{I}$.

We can now recall the definition of slice regularity.

Definition 2.1. Let $\Omega$ be a domain in $\mathbb{H}$. A function $f: \Omega \rightarrow \mathbb{H}$ is called (left) slice regular if, for all $I \in \mathbb{S}$, its restriction $f_{I}$ to $\Omega_{I}$ is holomorphic, i.e., it has continuous partial derivatives and satisfies

$$
\bar{\partial}_{I} f(x+y I):=\frac{1}{2}\left(\frac{\partial}{\partial x}+I \frac{\partial}{\partial y}\right) f_{I}(x+y I)=0
$$


for all $x+y I \in \Omega_{I}$.

Definition 2.2. Let $f: \mathbb{B} \rightarrow \mathbb{H}$ be a slice regular function. For each $I \in \mathbb{S}$, the $I$-derivative of $f$ at $q=x+y I$ is defined by

$$
\partial_{I} f(x+y I):=\frac{1}{2}\left(\frac{\partial}{\partial x}-I \frac{\partial}{\partial y}\right) f_{I}(x+y I)
$$

on $\mathbb{B}_{I}$. The slice derivative of $f$ is the function $f^{\prime}$ defined by $\partial_{I} f$ on $\mathbb{B}_{I}$ for all $I \in \mathbb{S}$.

All slice regular functions on $\mathbb{B}$ can be expressed as power series.

Theorem 2.3. Let $f: \mathbb{B} \rightarrow \mathbb{H}$ be a slice regular function. Then

$$
f(q)=\sum_{n=0}^{\infty} q^{n} a_{n}, \quad \text { with } a_{n}=\frac{f^{(n)}(0)}{n !}
$$

for all $q \in \mathbb{B}$.

In fact, there are some limitations in the expansion given in Theorem 2.3. Hence, a new type of expansion occurs in 47.

Definition 2.4. Let $f, g: \mathbb{B} \rightarrow \mathbb{H}$ be two slice regular functions of the form

$$
f(q)=\sum_{n=0}^{\infty} q^{n} a_{n}, \quad g(q)=\sum_{n=0}^{\infty} q^{n} b_{n} .
$$

The regular product (or $*$-product) of $f$ and $g$ is the slice regular function defined by

$$
f * g(q)=\sum_{n=0}^{\infty} q^{n}\left(\sum_{k=0}^{n} a_{k} b_{n-k}\right)
$$

Definition 2.5. Let $f(q)=\sum_{n=0}^{\infty} q^{n} a_{n}$ be a slice regular function on $\mathbb{B}$. We define the regular conjugate of $f$ as

$$
f^{c}(q)=\sum_{n=0}^{\infty} q^{n} \bar{a}_{n}
$$

and the symmetrization of $f$ as

$$
f^{s}(q)=f * f^{c}(q)=f^{c} * f(q)=\sum_{n=0}^{\infty} q^{n}\left(\sum_{k=0}^{n} a_{k} \bar{a}_{n-k}\right) .
$$

Both $f^{c}$ and $f^{s}$ are slice regular functions on $\mathbb{B}$.

Let $\mathcal{Z}_{f^{s}}$ denote the zero set of the symmetrization $f^{s}$ of $f$.

Definition 2.6. Let $f$ be a slice regular function on $\mathbb{B}$. If $f$ does not vanish identically, its regular reciprocal is the function defined by

$$
f^{-*}(q):=f^{s}(q)^{-1} f^{c}(q)
$$

which is slice regular on $\mathbb{B} \backslash \mathcal{Z}_{f s}$. 
Proposition 2.7. Let $f$ and $g$ be slice regular functions on $\mathbb{B}$. Then for all $q \in \mathbb{B} \backslash \mathcal{Z}_{f}$,

$$
f^{-*} * g(q)=f\left(T_{f}(q)\right)^{-1} g\left(T_{f}(q)\right),
$$

where $T_{f}: \mathbb{B} \backslash \mathcal{Z}_{f^{s}} \rightarrow \mathbb{B} \backslash \mathcal{Z}_{f}$ is defined by $T_{f}(q)=f^{c}(q)^{-1} q f^{c}(q)$. Furthermore, $T_{f}$ and $T_{f^{c}}$ are mutual inverses so that $T_{f}$ is a diffeomorphism.

In one complex variable, the starlike function of order $\alpha \in[0,1]$ was first introduced by Robertson [43. Now we introduce its corresponding version for slice regular functions.

Definition 2.8. Let $\alpha<1$. We shall say that $f$ is a slice starlike function of order $\alpha$ in the unit ball $\mathbb{B}$ if the slice regular function $f \in \mathcal{S}$ satisfies

$$
\operatorname{Re}\left(f(q)^{-1} q f^{\prime}(q)\right)>\alpha, \quad q \in \mathbb{B} \backslash\{0\} .
$$

Specially, we shall say that $f$ is a slice starlike function for $\alpha=0$.

Let $\mathcal{S}^{*}(\alpha)$ denote the class of slice starlike function of order $\alpha$ and write $\mathcal{S}^{*}$ instead when $\alpha=0$.

With the concept of slice starlike functions, we can introduce the concept of slice close-to-convex functions given by

$$
\mathcal{C}=\left\{f: \mathbb{B} \rightarrow \mathbb{H}: \exists h \in \mathcal{S}^{*} \text { such that } \operatorname{Re}\left(h(q)^{-1} q f^{\prime}(q)\right)>0 \text { on } \mathbb{B}\right\} .
$$

Note that the inclusion $\mathcal{S}^{*} \subset \mathcal{C}$ holds.

In one complex variable case, a holomorphic function $f$ in $\mathcal{C}$ is the so-called close-to-convex function introduced by Kaplan. In [32, Theorem 1], it was proven that every close-to-convex function is injective which satisfies naturally the Bieberbach conjecture. In fact, this result was proved early by Reade in 1955 (see e.g., [34, 38]).

Note that we have omitted the word "left" for simplicity in the article. Certainly, the right slice regular functions have completely analogous theories. This observation shall be used in the proof of Theorem 3.11

\section{EXAMPLES AND LEMMAS}

In this section, we shall offer some examples in the function class $\mathcal{C}$ and give some useful lemmas to prove Theorems 1.1 and 1.2 In addition, we establish a Rogosinski lemma for slice regular functions.

Example 3.1. Let $f(q)=q+\sum_{n=2}^{+\infty} q^{n} a_{n}$ be a slice regular function on $\mathbb{B}$ such that $\sum_{n=2}^{+\infty} n\left|a_{n}\right| \leq 1$. Then $f$ is injective on $\mathbb{B}$ and is in $\mathcal{S}^{*}$.

Proof. In fact, for any integer $n \geq 2$,

$$
\left|q_{1}^{n}-q_{2}^{n}\right|<n\left|q_{1}-q_{2}\right|, \quad \forall q_{1}, q_{2} \in \mathbb{B}, q_{1} \neq q_{2} .
$$

For $n=2$, there holds that

$$
q_{1}^{2}-q_{2}^{2}=q_{1}\left(q_{1}-q_{2}\right)+\left(q_{1}-q_{2}\right) q_{2} .
$$

Then, for $q_{1}, q_{2} \in \mathbb{B}$ with $q_{1} \neq q_{2}$,

$$
\left|q_{1}^{2}-q_{2}^{2}\right| \leq\left|q_{1}\left(q_{1}-q_{2}\right)\right|+\left|\left(q_{1}-q_{2}\right) q_{2}\right|<2\left|q_{1}-q_{2}\right| .
$$

By induction, we can readily obtain (3.1) for the general case $n \geq 2$. 
By the assumption of $f$ and (3.1), it follows that, for $q_{1}, q_{2} \in \mathbb{B}$ with $q_{1} \neq q_{2}$,

$$
\left|f\left(q_{1}\right)-f\left(q_{2}\right)\right| \geq\left|q_{1}-q_{2}\right|-\sum_{n=2}^{+\infty}\left|q_{1}^{n}-q_{2}^{n}\right|\left|a_{n}\right|>\left|q_{1}-q_{2}\right|\left(1-\sum_{n=2}^{+\infty} n\left|a_{n}\right|\right) \geq 0
$$

which shows that $f$ is injective on $\mathbb{B}$.

Now let us show $f \in \mathcal{S}^{*}$. Under the assumption of $f$, we have

$$
\left|q f^{\prime}(q)-f(q)\right| \leq \sum_{n=2}^{+\infty}|q|^{n}(n-1)\left|a_{n}\right|<|q|-\sum_{n=2}^{+\infty}|q|^{n}\left|a_{n}\right| \leq|f(q)|, \quad \forall q \in \mathbb{B} \backslash\{0\} .
$$

Hence, $\mathcal{Z}_{f}=\{0\}$ and

$$
\left|f(q)^{-1} q f^{\prime}(q)-1\right|=\left|f(q)^{-1}\right|\left|q f^{\prime}(q)-f(q)\right|<1, \quad \forall q \in \mathbb{B} \backslash\{0\},
$$

which implies naturally that $\operatorname{Re}\left(f(q)^{-1} q f^{\prime}(q)\right)>0$ on $\mathbb{B}$. Thus $f \in \mathcal{S}^{*}$, as desired.

See 26] for the complex case of Example 3.1. In order to introduce other important examples, we need to recall the concept of starlike function and a well-known analytical characterization of starlikeness (see e.g., [28, p. 36]). Let $\Omega \subset \mathbb{R}^{n}$. The set $\Omega$ is called starlike with respect to the origin 0 , if $t \Omega \subset \Omega$ for all $t \in[0,1]$. Let $F$ be holomorphic on $\mathbb{D}$. We say that $F$ is a starlike function on $\mathbb{D}$ with respect to 0 if $F$ is injective on $\mathbb{D}$ and the image $F(\mathbb{D})$ is starlike with respect to 0 .

Theorem 3.2. Let $F: \mathbb{D} \rightarrow \mathbb{C}$ be a holomorphic function with $F(0)=0$ and $F^{\prime}(0)=1$. Then $F$ is starlike with respect to 0 if and only if $\operatorname{Re} \frac{z F^{\prime}(z)}{F(z)}>0$ on $\mathbb{D}$.

We recall also a so-called convex combination identity for slice regular functions which was used to establish sharp growth and distortion theorems for a subclass of slice regular functions (see [41] or [42]).

Lemma 3.3. Let $f$ be a slice regular function on $\mathbb{B}$ such that $f\left(\mathbb{B}_{I}\right) \subseteq \mathbb{C}_{I}$ for some $I \in \mathbb{S}$. Then for every $r e^{J \theta} \in \mathbb{B}$ with $J \in \mathbb{S}$,

$$
\left|f\left(r e^{J \theta}\right)\right|^{2}=\frac{1+\langle I, J\rangle}{2}\left|f\left(r e^{I \theta}\right)\right|^{2}+\frac{1-\langle I, J\rangle}{2}\left|f\left(r e^{-I \theta}\right)\right|^{2}
$$

where $\langle I, J\rangle=-\operatorname{Re}(I J) \in[-1,1]$.

From Theorem 3.2 Lemmas 3.3 and 3.7 below, one can show that

Example 3.4. Fix $I \in \mathbb{S}$. Let $F(z)=z+\sum_{n=2}^{+\infty} z^{n} a_{n}, a_{n} \in \mathbb{C}_{I}$ for $n=2,3,4 \ldots$ be a starlike function on $\mathbb{B}_{I} \simeq \mathbb{D}$. Then $f(q)=q+\sum_{n=2}^{+\infty} q^{n} a_{n} \in \mathcal{S}^{*}$.

Example 3.5. Let $f \in \mathcal{S}$ be such that

$$
\operatorname{Re}\left((f(q)-f(-q))^{-1} q f^{\prime}(q)\right)>0, \quad \forall q \in \mathbb{B} .
$$

Then $f \in \mathcal{C}$.

Proof. From (3.7) below, it follows that

$$
r \frac{\partial}{\partial r} \log |f(q)-f(-q)|=\operatorname{Re}\left((f(q)-f(-q))^{-1} q\left(f^{\prime}(q)+f^{\prime}(-q)\right)\right),
$$

for $q=r u \in \mathbb{B} \backslash\{0\}$ with $r=|q|$.

Then condition (3.3) and Lemma 3.7 imply that $(f(q)-f(-q)) / 2 \in \mathcal{S}^{*}$, thus $f \in \mathcal{C}$ by definition. 
To show Lemma 3.7 we prove the following useful result which reveals the close relation between the usual and regular multiplication.

Proposition 3.6. Let $f, g$ be two slice regular functions on $\mathbb{B}$ such that $\mathcal{Z}_{f}=\varnothing$. Then we have

$$
\operatorname{Re}\left(f(q)^{-1} g(q)\right)>0 \text { on } \mathbb{B} \Leftrightarrow \operatorname{Re}\left(f(q)^{-*} * g(q)\right)>0 \text { on } \mathbb{B},
$$

and

$$
|g(q)|<|f(q)| \text { on } \mathbb{B} \Leftrightarrow\left|f(q)^{-*} * g(q)\right|<1 \text { on } \mathbb{B} \text {. }
$$

Proof. We only prove the first claim (3.4). The second can be obtained by the same strategy. Note that $\mathcal{Z}_{f}=\varnothing$ if and only if $\mathcal{Z}_{f^{c}}=\varnothing$ for any slice regular function on $\mathbb{B}$. Assume that $\operatorname{Re}\left(f(q)^{-1} g(q)\right)>0$ on $\mathbb{B}$. From the assumption on the functions $f$ and $g$, we have that, by Proposition 2.7

$$
f^{-*} * g(q)=f\left(T_{f}(q)\right)^{-1} g\left(T_{f}(q)\right), \quad \forall q \in \mathbb{B},
$$

where $T_{f}(q)=f^{c}(q)^{-1} q f^{c}(q) \in \mathbb{B}$. This implies that $\operatorname{Re}\left(f(q)^{-*} * g(q)\right)>0$ on $\mathbb{B}$.

Conversely, the condition $\operatorname{Re}\left(f(q)^{-*} * g(q)\right)>0$ on $\mathbb{B}$ implies that $\operatorname{Re}\left(f\left(T_{f}(q)\right)^{-1} g\left(T_{f}(q)\right)\right)>0$ on $\mathbb{B}$ by (3.6). Then the desired result follows from the fact that $T_{f} \circ T_{f^{c}}(q)=q$ for all $q \in \mathbb{B}$.

Lemma 3.7. Let $f$ be a slice regular function on $\mathbb{B}$ such that $\mathcal{Z}_{f}=\{0\}$ and $f^{\prime}(0)=1$. Then the following statements are equivalent:

(1) $\operatorname{Re}\left(f(q)^{-1} q f^{\prime}(q)\right)>\alpha$ on $\mathbb{B} \backslash\{0\}$;

(2) $\operatorname{Re}\left(f(q)^{-*} *\left(q f^{\prime}(q)\right)\right)>\alpha$ on $\mathbb{B} \backslash\{0\}$;

(3) For any $u \in \partial \mathbb{B}$, the function $M(r)=|f(r u)| / r^{\alpha}$ is strictly increasing on $(0,1)$.

Proof. (1) $\Leftrightarrow(2)$ : It is trivial by Proposition 3.6.

$(1) \Rightarrow(3)$ : Notice that, for any slice regular function $f(q)$ on $\mathbb{B}$ with $q=r u=|q| u \in \mathbb{B}$, there holds that

Then, for $q \in \mathbb{B} \backslash\{0\}$,

$$
r \frac{\partial}{\partial r} f(q)=q f^{\prime}(q)
$$

$$
r \frac{\partial}{\partial r} \log |f(q)|=\frac{r}{|f(q)|^{2}} \operatorname{Re}\left(\frac{\partial}{\partial r} f(q) \overline{f(q)}\right)=\operatorname{Re}\left(f(q)^{-1} q f^{\prime}(q)\right)>\alpha .
$$

Integrating the above inequality from $r_{1}$ to $r_{2}$ with $0<r_{1}<r_{2}<1$ gives that

$$
\frac{\left|f\left(r_{2} u\right)\right|}{\left|f\left(r_{1} u\right)\right|}>\frac{r_{2}^{\alpha}}{r_{1}^{\alpha}}
$$

which implies statement (3).

$(3) \Rightarrow(1)$ : Fix $u \in \partial \mathbb{B}$. Under the condition of $(3)$, we have, for $q=r u, r \in(0,1)$,

$$
\frac{\partial}{\partial r} \frac{|f(r u)|}{r^{\alpha}} \geq 0
$$

which implies

$$
r \frac{\partial}{\partial r} \log |f(r u)| \geq \alpha .
$$

This together with (3.7) shows that $\operatorname{Re}\left(f(q)^{-1} q f^{\prime}(q)\right) \geq \alpha$ for all $r \in(0,1)$. Since $u \in \partial \mathbb{B}$ is arbitrary, it follows that

$$
\operatorname{Re}\left(f(q)^{-1} q f^{\prime}(q)\right) \geq \alpha, \quad \forall q \in \mathbb{B} \backslash\{0\}
$$


Let us show that inequality (3.8) is strict. Consider the slice regular function

$$
h(q)= \begin{cases}f(q)^{-*} *\left(q f^{\prime}(q)\right) & \text { if } q \in \mathbb{B} \backslash\{0\}, \\ 1, & \text { if } q=0 .\end{cases}
$$

By Proposition 3.6 (3.8) implies that

$$
\operatorname{Re} h(q) \geq \alpha, \quad \forall q \in \mathbb{B} .
$$

If equality occurs in (3.8) for some $q_{0} \in \mathbb{B}$, then $\operatorname{Re} h\left(\widetilde{q_{0}}\right)=\alpha$ for $\widetilde{q_{0}}=f\left(q_{0}\right)^{-1} q_{0} f\left(q_{0}\right) \in \mathbb{B}$. By applying the maximum principle (see [22, Theorem 7.13]) for the real part of a slice regular function to $-h$, we see that $h$ is constant on $\mathbb{B}$. Whence $f(q)=q f^{\prime}(q)$ on $\mathbb{B}$, which forces that $f$ is an identity on $\mathbb{B}$. Thus, for $\alpha<1$, (3.8) is strict.

Hence statements (1) and (3) are equivalent.

Note that $\mathcal{S}^{*}(\alpha)=\emptyset$ if $\alpha \geq 1$ from Lemma 3.7

As a byproduct, by Theorem 3.2 and Lemma 3.7 we obtain a characterization for starlike functions in one complex variable case.

Corollary 3.8. Let $F$ be holomorphic on $\mathbb{D}$ such that $F(0)=0$ and $F^{\prime}(0)=1$. Then the following statements are equivalent.

(1) $F$ is starlike with respect to 0 ;

(2) $\left.\operatorname{Re}\left(F(z)^{-1} z F^{\prime}(z)\right)\right)>0$ on $\mathbb{D}$;

(3) for any $\theta \in[0,2 \pi)$, the function $M(r)=\left|F\left(r e^{i \theta}\right)\right|$ is strictly increasing on $[0,1)$.

Remark 3.9. Note that condition (2) in Corollary 3.8 would imply that $F$ is injective on $\mathbb{D}$. From the formula

$$
\operatorname{Re}\left(\frac{z F^{\prime}(z)}{F(z)}\right)=\frac{\partial}{\partial \theta} \arg F\left(r e^{i \theta}\right), \quad z=r e^{i \theta}
$$

it follows that the argument of $F\left(r e^{i \theta}\right)$ increases monotonically for $\theta$. Thus $F$ is injective on $|z|=r<1$. By the principle of univalence on the boundary, we can conclude that $F$ is injective on $|z| \leq r$. Since $r<1$ is arbitrary, $F$ is injective on $\mathbb{D}$ (see e.g., [28, p. 38]). However, this approach is not suitable for the quaternionic setting.

Now we state without proof a variant of the Schwarz lemma for slice regular functions in [19], Theorem 4.1] which is useful in the sequel.

Theorem 3.10. If $f: \mathbb{B} \rightarrow \mathbb{B}$ be slice regular such that $f(0)=f^{\prime}(0)=\ldots=f^{(m-1)}(0)=0 \quad(m \geq 1)$. Then

$$
|f(q)| \leq|q|^{m}, \quad \forall q \in \mathbb{B},
$$

and

$$
\left|f^{(m)}(0)\right| \leq m !
$$

Both inequalities are strict (except $q=0$ ) unless $f(q)=q^{m} u$ for some $u \in \partial \mathbb{B}$.

In fact, we can establish a sharpened form of the Schwarz lemma for slice regular functions. Its complex version is due to Rogosinski (see e.g., [13, p. 200]).

For $q_{0} \in \mathbb{H}$, denote $B\left(q_{0}, r\right)=\left\{q \in \mathbb{H}:\left|q-q_{0}\right|<r\right\}$. 
Theorem 3.11. Let $q_{0}, b \in \mathbb{B}$. For the set of all slice regular functions $f: \mathbb{B} \rightarrow \mathbb{B}$ with $f(0)=0$ and $f^{\prime}(0)=b$, the range of values of $f\left(q_{0}\right)$ is the closed ball $\overline{B(c, r)}$, where

$$
c=\frac{q_{0} b\left(1-\left|q_{0}\right|^{2}\right)}{1-\left|q_{0} b\right|^{2}}, \quad r=\frac{\left|q_{0}\right|^{2}\left(1-|b|^{2}\right)}{1-\left|q_{0} b\right|^{2}} .
$$

Proof. For $b=0$, the assertion is trivial. Now we consider the case for $b \neq 0$. Denote the slice regular function $g(q)=q^{-1} f(q)$ on $\mathbb{B}$ with $g(0)=b$ and $|g(q)| \leq 1$ for all $q \in \mathbb{B}$ by the maximum modulus principle for slice regular functions (cf. [22, Theorem 7.1]). Denote the slice regular function

$$
h(q)=(1-g(q) \bar{b})^{-*} *(b-g(q)),
$$

with $h(0)=0$ and $|h(q)|<1$ for all $q \in \mathbb{B}$ by Proposition 2.7 From Theorem 3.10 it follows that

$$
|h(q)| \leq|q|, \quad \forall q \in \mathbb{B} .
$$

That is, by Proposition 2.7

$$
\left|\left(1-g \circ T_{(1-g \bar{b})^{c}}(q) \bar{b}\right)^{-1}\left(b-g \circ T_{(1-g \bar{b})^{c}}(q)\right)\right| \leq|q|, \quad \forall q \in \mathbb{B} .
$$

Since $T_{(1-g \bar{b})^{c}} \circ T_{1-g \bar{b}}(q)=q$ and $\left|T_{1-g \bar{b}}(q)\right|=|q|$, we obtain

$$
\left|(1-g(q) \bar{b})^{-1}(b-g(q))\right| \leq|q|, \quad \forall q \in \mathbb{B} .
$$

Equivalently,

A direct computation shows that

$$
\frac{|f(q)-q b|}{\left|f(q)-q \bar{b}^{-1}\right|} \leq|b q|, \quad \forall q \in \mathbb{B} .
$$

$$
\left|f(q)-\frac{q b\left(1-|q|^{2}\right)}{1-|q b|^{2}}\right| \leq \frac{|q|^{2}\left(1-|b|^{2}\right)}{1-|q b|^{2}}, \quad \forall q \in \mathbb{B} .
$$

Hence $f\left(q_{0}\right) \in \overline{B(c, r)}$ with $c$ and $r$ as desired.

To see that $\overline{B(c, r)}$ is covered, let $p \in \overline{\mathbb{B}}$ and set the slice regular function

$$
f(q)=q(1-q|b| p)^{-*} *(|b|-q p) \frac{b}{|b|}, \quad \forall q \in \mathbb{B} .
$$

It is easy to see that $f(0)=0$ and $f^{\prime}(0)=b$. Now we show that $|f(q)|<1$ for all $q \in \mathbb{B}$. To this end, we only prove that

$$
\left|(1-q|b| p)^{-*} *(|b|-q p)\right|<1, \quad \forall q \in \mathbb{B} .
$$

Note that

$$
\left(1-|b|^{2}\right)\left(1-|q p|^{2}\right)>0, \quad \forall q \in \mathbb{B},
$$

that is to say

$$
|| b|-q p|<|1-q| b|p|, \quad \forall q \in \mathbb{B} .
$$

Therefore, [3.9) holds by Proposition 3.6

After a lengthy but direct calculation, there holds that

$$
f(q)=\frac{q b\left(1-|q|^{2}\right)}{1-|q b|^{2}}+\frac{q^{2}\left(1-|b|^{2}\right)}{1-|q b|^{2}} \phi(p) \frac{b}{|b|},
$$

where

$$
\phi(p)=\bar{q}|b|-\left(1-|q b|^{2}\right) \sum_{n=1}^{+\infty}(|b| q)^{n-1} p^{n}=(|b| \bar{q}-p) *_{r}(1-q|b| p)^{-*_{r}}
$$


is a right slice regular Möbious transform of $\mathbb{B}$.

Since $p \in \overline{\mathbb{B}}$ is arbitrary, it follows that $\phi(\overline{\mathbb{B}})=\overline{\mathbb{B}}$.

Note that, for any $u \in \partial \mathbb{B}$,

$$
\{q u: q \in \overline{\mathbb{B}}\}=\overline{\mathbb{B}}
$$

Hence the closed ball $\overline{B(c, r)}$ is covered. The proof is complete.

From Theorem 3.11 and its proof we obtain a sharper Schwarz lemma.

Corollary 3.12. Let $f$ be a slice regular function on $\mathbb{B}$ with $f(0)=0$ and $|f(q)|<1$ for all $q \in \mathbb{B}$. Then

$$
|q| \frac{\left|f^{\prime}(0)\right|-|q|}{1-\left|q f^{\prime}(0)\right|} \leq|f(q)| \leq|q| \frac{|q|+\left|f^{\prime}(0)\right|}{1+\left|q f^{\prime}(0)\right|}, \quad \forall q \in \mathbb{B} .
$$

Equality holds at some point $q_{0} \neq 0$ if and only if $f(q)=q \varphi_{a}(q) u$ for some $a \in \mathbb{B}, u \in \partial \mathbb{B}$.

As an important generalization of the Schwarz lemma, the classical Schwarz-Pick lemma states that if $F$ is a holomorphic self-mapping on $\mathbb{D}$, then

$$
\left|F^{\prime}(z)\right| \leq \frac{1-|F(z)|^{2}}{1-|z|^{2}}, \quad \forall z \in \mathbb{D}
$$

However, this classical version fails even for the slice regular automorphisms. Indeed, we take the slice regular Möbius transformation (see [46, Theorem 7.6])

$$
\varphi_{a}(q)=(1-q \bar{a})^{-*} *(a-q)=a-\left(1-|a|^{2}\right) \sum_{n=1}^{+\infty} q^{n} \bar{a}^{n-1} .
$$

Pick $a=\frac{i}{2}$ and $q_{0}=\frac{j}{2}$. By direct computation, we have

$$
\varphi_{a}\left(q_{0}\right)=\frac{2}{5}(i-j), \quad \varphi_{a}^{\prime}\left(q_{0}\right)=-\frac{204}{225}-\frac{96}{225} k,
$$

so that

$$
\left|\varphi_{a}^{\prime}\left(q_{0}\right)\right|=\sqrt{\frac{50832}{50625}}>\frac{1-\left|\varphi_{a}\left(q_{0}\right)\right|^{2}}{1-\left|q_{0}\right|^{2}}=\frac{68}{75} .
$$

Fortunately, a variant of the Schwarz-Pick lemma for self-mapping of $\mathbb{B}$ was established in [5] As a special case, one can obtain a coefficient estimate.

Lemma 3.13. Let $f$ be a slice regular function on $\mathbb{B}$ such that $|f(q)| \leq 1$ for all $q \in \mathbb{B}$. Then

$$
\left|f^{\prime}(0)\right| \leq 1-|f(0)|^{2}
$$

Equality holds in (3.11) if and only if $f$ is of the form

$$
f(q)=(1-q \bar{a})^{-*} *(a-q) u, \quad \forall q \in \mathbb{B},
$$

for some $a \in \overline{\mathbb{B}}, u \in \partial \mathbb{B}$. 


\section{Coefficient Estimates}

As in the complex holomorphic case, let $\mathcal{P}$ denote the class of slice regular functions $p$ on $\mathbb{B}$ such that $p(0)=1$ and $\operatorname{Re} p(q)>0$ on $\mathbb{B}$. The function class $\mathcal{P}$ is usually called the Carathéodory class.

To prove Theorems 1.1 and 1.2 we shall first present a coefficient estimate for the class $\mathcal{P}$ based on Lemma 3.13

Theorem 4.1. Let $f(q)=1+\sum_{n=1}^{\infty} q^{n} a_{n}$ be a function in $\mathcal{P}$. Then

$$
\left|a_{2}-\frac{a_{1}^{2}}{2}\right| \leq 2-\frac{\left|a_{1}\right|^{2}}{2} .
$$

Equality holds in 4.11) if and only if

$$
f(q)=\left(q \varphi_{a}(q) u+1\right) *\left(1-q \varphi_{a}(q) u\right)^{-*},
$$

for some $a \in \overline{\mathbb{B}}$ and $u \in \partial \mathbb{B}$.

Proof. Define

$$
g(q)= \begin{cases}q^{-1}(f(q)+1)^{-*} *(f(q)-1), & \text { if } q \in \mathbb{B} \backslash\{0\} \\ a_{1} / 2, & \text { if } q=0\end{cases}
$$

From the assumption of $f$, we have

$$
\left|(f(q)+1)^{-1}(f(q)-1)\right|<1, \quad \forall q \in \mathbb{B},
$$

then, by Proposition [3.6 the function $h(q)=(f(q)+1)^{-*} *(f(q)-1)$ is slice regular on $\mathbb{B}$ with $h(0)=0, h^{\prime}(0)=a_{1} / 2$ and $|h(q)|<1$ for all $q \in \mathbb{B}$. By the maximum modulus principle for slice regular functions (cf. [22, Theorem 7.1]), we obtain that the function $g$ is a slice regular function on $\mathbb{B}$ with $g^{\prime}(0)=a_{2} / 2-a_{1}^{2} / 4$ and $|g(q)| \leq 1$ for all $q \in \mathbb{B}$. From Lemma 3.13 (4.1) follows and equality holds in (4.1) if and only if $g(q)=\varphi_{a}(q) u$ for some $a \in \overline{\mathbb{B}}$ and $u \in \partial \mathbb{B}$. By simple calculations, we deduce that

$$
f(q)=\left(q \varphi_{a}(q) u+1\right) *\left(1-q \varphi_{a}(q) u\right)^{-*} .
$$

Now the proof is complete.

As a direct consequence, we obtain all coefficient estimates for the Carathéodory class $\mathcal{P}$ applying the approach of finite average as in the case of one complex variable. Now let us recall this result in 39 .

Theorem 4.2. Let $f(q)=1+\sum_{n=1}^{\infty} q^{n} a_{n}$ be a slice regular function in $\mathcal{P}$. Then

$$
\frac{1-|q|}{1+|q|} \leq \operatorname{Re} f(q) \leq|f(q)| \leq \frac{1+|q|}{1-|q|}, \quad \forall q \in \mathbb{B},
$$

and

$$
\left|a_{n}\right| \leq 2, \quad n=1,2, \ldots
$$

Moreover, $\left|a_{1}\right|=2$ or equality holds for the first or third inequality in (4.2) at some $q_{0} \neq 0$ if and only if

$$
f(q)=(1-q u)^{-*} *(1+q u), \quad \forall q \in \mathbb{B},
$$

for some $u \in \partial \mathbb{B}$. 
Now we can prove our main coefficient estimates.

Proof of Theorem 1.1. Define

$$
p(q)= \begin{cases}\left(q^{-1} f(q)\right)^{-*} * f^{\prime}(q) & \text { if } q \in \mathbb{B} \backslash\{0\}, \\ 1, & \text { if } q=0,\end{cases}
$$

which belongs to $\mathcal{P}$ by Lemma 3.7 Set $p(z)=1+q p_{1}+\cdots$. Applying Theorem 4.2 to function $p$, we have $\left|p_{n}\right| \leq 2$ for all $n \geq 1$. Comparing the coefficients in the power series of $f^{\prime}(q)$ and $q^{-1} f(q) * p(q)$, we have

$$
n a_{n}=p_{n-1}+a_{2} p_{n-2}+\cdots+a_{n-1} p_{1}+a_{n}, \quad n=2,3, \ldots
$$

By induction, we can obtain $\left|a_{n}\right| \leq n$ for all $n=2,3, \ldots$.

It is easy to see that if $\left|a_{n}\right|=n$ for a given $n$, then the above arguments imply that $\left|p_{1}\right|=\left|a_{2}\right|=2$, and thus, by Theorem 4.2 , $p$ is of the form

$$
p(q)=(1-q u)^{-*} *(1+q u)=1+\sum_{n=1}^{+\infty} q^{n} 2 u^{n}, \quad \forall q \in \mathbb{B}
$$

for some $u \in \partial \mathbb{B}$. Hence, by (4.4), we have

$$
(n-1) a_{n}=2\left(u^{n-1}+a_{2} u^{n-2}+\cdots+a_{n-1} u\right), \quad n=2,3, \ldots,
$$

which leads to that $a_{n}=n u^{n-1}$ for all $n=2,3, \ldots$, as desired.

Note that Theorem 1.1 generalizes Theorem 2.2.16 in 28] to the non-commutative algebra. Nevanlinna established the complex version of Theorem 1.1 for normalized starlike functions in 1920. In fact, the Bieberbach conjecture over quaternions still holds for slice close-to-convex functions.

Theorem 4.3. Let $f(q)=q+\sum_{n=2}^{+\infty} q^{n} a_{n} \in \mathcal{C}$. Then

$$
\left|a_{n}\right| \leq n, \text { for all } n=2,3, \ldots
$$

Equality $\left|a_{n}\right|=n$ for a given $n \geq 2$ holds if and only if

$$
f(q)=q(1-q u)^{-* 2}, \quad \forall q \in \mathbb{B},
$$

for some $u \in \partial \mathbb{B}$.

Proof. By definition, there exists a function $h=q+q^{2} h_{2}+\cdots \in \mathcal{S}^{*}$ such that

$$
p(q)=\left(q^{-1} h(q)\right)^{-*} * f^{\prime}(q)=1+q p_{1}+q^{2} p_{2}+\cdots, \quad q \in \mathbb{B},
$$

which belongs to $\mathcal{P}$ by Lemma 3.7

From (4.5), we have

$$
f^{\prime}(q)=q^{-1} h(q) * p(q), \quad q \in \mathbb{B} .
$$

Comparing the coefficients in the power series above, we have

$$
n a_{n}=p_{n-1}+h_{2} p_{n-2}+\cdots+h_{n-1} p_{1}+h_{n}, \quad n=2,3, \ldots
$$

Applying Theorem 4.2 to function $p$, we obtain that $\left|p_{n}\right| \leq 2$ for all $n \geq 1$. From the assumption of $h$, by Theorem 1.1 we have $\left|h_{n}\right| \leq n$ for all $n=2,3, \ldots$. Combining these two estimates with (4.6), it follows that $\left|a_{n}\right| \leq n$ for all $n=2,3, \ldots$. 
Following the proof in Theorem 1.1 the condition of equality can be obtained. This completes the proof.

Proof of Theorem 1.2. Let us consider the function

$$
g(q)= \begin{cases}\left(q f^{\prime}(q)+f(q)\right)^{-*} *\left(q f^{\prime}(q)-f(q)\right) & \text { if } q \in \mathbb{B} \backslash\{0\} \\ 0, & \text { if } q=0 .\end{cases}
$$

Then it easy to see that $g$ is slice regular on $\mathbb{B}$ with

$$
g_{1}=g^{\prime}(0)=\frac{a_{2}}{2}, \quad g_{2}=\frac{g^{\prime \prime}(0)}{2}=a_{3}-\frac{3}{4} a_{2}^{2} .
$$

From the assumption of $f$, we have

$$
\left|\left(q f^{\prime}(q)+f(q)\right)^{-1}\left(q f^{\prime}(q)-f(q)\right)\right|<1, \quad \forall q \in \mathbb{B} .
$$

By Lemma 3.7 $|g(q)|<1$ for all $q \in \mathbb{B}$, which gives that, by Lemma 3.13

$$
\left|g_{2}-\lambda g_{1}^{2}\right| \leq 1-\left|g_{1}\right|^{2}+|\lambda|\left|g_{1}\right|^{2} \leq \max \{1,|\lambda|\}
$$

that is

$$
\left|a_{3}-\frac{3}{4} a_{2}^{2}-\lambda \frac{1}{4} a_{2}^{2}\right| \leq \max \{1,|\lambda|\}
$$

Hence,

$$
\left|a_{3}-\lambda a_{2}^{2}\right| \leq \max \{1,|4 \lambda-3|\} .
$$

The sharpness of the estimate can be easily checked from the function given in the theorem. Now the proof is complete.

\section{Growth And Distortion Theorems}

In this section, we shall study the growth and distortion theorems for slice regular functions.

From Corollary 3.12 , we first present a growth theorem for the Carathéodory class $\mathcal{P}$ which is an improved version of Theorem 4.2

Theorem 5.1. Let $p(q)=1+\sum_{n=1}^{\infty} q^{n} a_{n}$ be a slice regular function in $\mathcal{P}$. Then

$$
\frac{1-|q|^{2}}{1+\left|a_{1} q\right|+|q|^{2}} \leq \operatorname{Re} p(q) \leq|p(q)| \leq \frac{1+\left|a_{1} q\right|+|q|^{2}}{1-|q|^{2}}, \quad \forall q \in \mathbb{B} .
$$

Moreover, equality holds for the first or third inequality in 5.1) at some $q_{0} \neq 0$ if and only if

$$
p(q)=\left(q \varphi_{a}(q) u+1\right) *\left(1-q \varphi_{a}(q) u\right)^{-*}, \quad \forall q \in \mathbb{B},
$$

for some $u \in \partial \mathbb{B}$.

Proof. Consider the slice regular function

$$
f(q)=(p(q)+1)^{-*} *(p(q)-1), \quad \forall q \in \mathbb{B} .
$$

Note that $f(0)=0, f^{\prime}(0)=a_{1} / 2$ and $|f(q)|<1$ for all $q \in \mathbb{B}$ by Proposition 3.6 then it follows that, by Corollary 3.12 ,

$$
|f(q)| \leq|q| \frac{|q|+\left|f^{\prime}(0)\right|}{1+\left|q f^{\prime}(0)\right|}=: \mu, \quad \forall q \in \mathbb{B} .
$$


By Proposition 2.7 it follows that

$$
\frac{|p(q)+1|}{|p(q)-1|} \leq \mu, \quad \forall q \in \mathbb{B} .
$$

i.e.,

$$
\left|p(q)-\frac{1+\mu^{2}}{1-\mu^{2}}\right| \leq \frac{2 \mu}{1-\mu^{2}}, \quad \forall q \in \mathbb{B} .
$$

Hence, (5.1) follows from

$$
|p(q)| \leq \frac{1+\mu^{2}}{1-\mu^{2}}+\frac{2 \mu}{1-\mu^{2}}=\frac{1+\left|q a_{1}\right|+|q|^{2}}{1-|q|^{2}}, \quad \forall q \in \mathbb{B} .
$$

and

$$
\operatorname{Re} p(q) \geq \frac{1+\mu^{2}}{1-\mu^{2}}-\frac{2 \mu}{1-\mu^{2}}=\frac{1-|q|^{2}}{1+\left|q a_{1}\right|+|q|^{2}}, \quad \forall q \in \mathbb{B} .
$$

If one of equalities above occurs for some point $q_{0} \neq 0$, then (5.2) turns into an equality at $\widetilde{q_{0}}=$ $T_{p^{c}+1}\left(q_{0}\right)=\left(p\left(q_{0}\right)+1\right)^{-1} q_{0}\left(p\left(q_{0}\right)+1\right)$. Whence Corollary 3.12 implies that

$$
f(q)=q \varphi_{a}(q) u, \quad \forall q \in \mathbb{B},
$$

for some $u \in \partial \mathbb{B}$. Thus

$$
p(q)=\left(q \varphi_{a}(q) u+1\right) *\left(1-q \varphi_{a}(q) u\right)^{-*}, \quad \forall q \in \mathbb{B},
$$

for some $u \in \partial \mathbb{B}$.

The converse part can be verified easily by direct calculations. Now the proof is complete.

Now we can establish the growth and distortion theorems for $\mathcal{S}^{*}$.

Theorem 5.2. Let $f(q)=q+\sum_{n=2}^{\infty} q^{n} a_{n} \in \mathcal{S}^{*}$. Then the following inequalities hold for all $q \in \mathbb{B}$

$$
\begin{gathered}
\frac{|q|}{1+\left|a_{2} q\right|+|q|^{2}} \leq|f(q)| \leq \frac{|q|}{1-|q|^{2}}\left(\frac{1+|q|}{1-|q|}\right)^{\frac{\left|a_{2}\right|}{2}} \\
\frac{1-|q|^{2}}{\left(1+\left|a_{2} q\right|+|q|^{2}\right)^{2}} \leq\left|f^{\prime}(q)\right| \leq \frac{1+\left|a_{2} q\right|+|q|^{2}}{\left(1-|q|^{2}\right)^{2}}\left(\frac{1+|q|}{1-|q|}\right)^{\frac{\left|a_{2}\right|}{2}} \\
\frac{1-|q|^{2}}{1+\left|a_{2} q\right|+|q|^{2}} \leq \frac{\left|q f^{\prime}(q)\right|}{|f(q)|} \leq \frac{1+\left|a_{2} q\right|+|q|^{2}}{1-|q|^{2}} .
\end{gathered}
$$

Proof. Following the proof of Theorem 1.1 and applying Theorem[5.1 we can obtain a combined growth and distortion theorem for $f \in \mathcal{S}^{*}$ and all $q \in \mathbb{B}$

$$
\frac{1-|q|^{2}}{1+\left|a_{2} q\right|+|q|^{2}} \leq \operatorname{Re}\left(f(q)^{-*} *\left(q f^{\prime}(q)\right)\right) \leq\left|f(q)^{-*} *\left(q f^{\prime}(q)\right)\right| \leq \frac{1+\left|a_{2} q\right|+|q|^{2}}{1-|q|^{2}} .
$$

Thus, by Proposition 2.7

$$
\frac{1-|q|^{2}}{1+\left|a_{2} q\right|+|q|^{2}} \leq \operatorname{Re}\left(f(q)^{-1} q f^{\prime}(q)\right) \leq \frac{\left|q f^{\prime}(q)\right|}{|f(q)|} \leq \frac{1+\left|a_{2} q\right|+|q|^{2}}{1-|q|^{2}}
$$

which implies two inequalities in (5.5).

From (3.7) and (5.6), we have

$$
\frac{1-r^{2}}{r\left(1+\left|a_{2}\right| r+r^{2}\right)} \leq \frac{\partial}{\partial r} \log |f(r u)| \leq \frac{1+\left|a_{2}\right| r+r^{2}}{r\left(1-r^{2}\right)}
$$


where $q=r u$ with $r=|q|$.

Integrating the above inequality along a radius from $r_{1}$ to $r_{2}$ with $0<r_{1}<r_{2}<1$ gives that

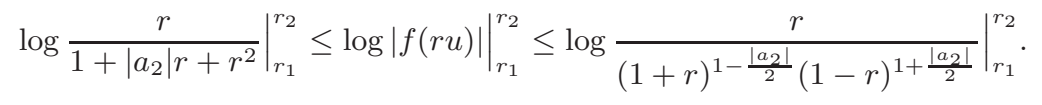

Equivalently,

$$
\frac{r_{2}}{1+\left|a_{2}\right| r_{2}+r_{2}^{2}} \varepsilon_{1}\left(r_{1}\right) \leq\left|f\left(r_{2} u\right)\right| \leq \frac{r_{2}}{1-r_{2}^{2}}\left(\frac{1+r_{2}}{1-r_{2}}\right)^{\frac{\left|a_{2}\right|}{2}} \varepsilon_{2}\left(r_{1}\right),
$$

where

$$
\varepsilon_{1}\left(r_{1}\right)=\left(1+\left|a_{2}\right| r_{1}+r_{1}^{2}\right) \frac{\left|f\left(r_{1} u\right)\right|}{r_{1}},
$$

and

$$
\varepsilon_{2}\left(r_{1}\right)=\left(1-r_{1}^{2}\right)\left(\frac{1-r_{1}}{1+r_{1}}\right)^{\frac{\left|a_{2}\right|}{2}} \frac{\left|f\left(r_{1} u\right)\right|}{r_{1}} .
$$

Letting $r_{1} \rightarrow 0^{+}$, (5.3) follows from

$$
\frac{r_{2}}{1+\left|a_{2}\right| r_{2}+r_{2}^{2}} \leq\left|f\left(r_{2} u\right)\right| \leq \frac{r_{2}}{1-r_{2}^{2}}\left(\frac{1+r_{2}}{1-r_{2}}\right)^{\frac{\left|a_{2}\right|}{2}} .
$$

Now (5.4) is a consequence of (5.3) and (5.5). The proof is complete.

Note that $\left|a_{2}\right| \leq 2$ provided $f(q)=q+\sum_{n=2}^{\infty} q^{n} a_{n} \in \mathcal{S}^{*}$. Hence we immediately obtain the following growth and distortion theorem for $\mathcal{S}^{*}$.

Theorem 5.3. Let $f \in \mathcal{S}^{*}$. Then the following inequalities hold for all $q \in \mathbb{B}$

$$
\begin{gathered}
\frac{|q|}{(1+|q|)^{2}} \leq|f(q)| \leq \frac{|q|}{(1-|q|)^{2}} ; \\
\frac{1-|q|}{(1+|q|)^{3}} \leq\left|f^{\prime}(q)\right| \leq \frac{1+|q|}{(1-|q|)^{3}} ; \\
\frac{1-|q|}{1+|q|} \leq \frac{\left|q f^{\prime}(q)\right|}{|f(q)|} \leq \frac{1+|q|}{1-|q|} .
\end{gathered}
$$

All of these estimates are sharp. For each $q \in \mathbb{B} \backslash\{0\}$, equality occurs if

$$
f(q)=q(1-q u)^{-* 2}, \quad q \in \mathbb{B},
$$

for some $u \in \partial \mathbb{B}$.

As a consequence of (5.8), Theorem 1.4 follows. Now we establish a more general version.

Theorem 5.4. Let $m$ be a positive integer and $f(q)=q+\sum_{n=m+1}^{+\infty} q^{n} a_{n}$ be a slice regular function on $\mathbb{B}$ such that $q f^{\prime}(q) \in \mathcal{S}^{*}$. Then

$$
\frac{1}{\left(1+|q|^{m}\right)^{2 / m}} \leq\left|f^{\prime}(q)\right| \leq \frac{1}{\left(1-|q|^{m}\right)^{2 / m}}, \quad \forall q \in \mathbb{B} .
$$


Proof. Denote the slice regular function

$$
g(q)=f^{\prime}(q)^{-*} *\left(q f^{\prime \prime}(q)\right), \quad \forall q \in \mathbb{B} .
$$

From the assumption of $f$, we have

$$
\operatorname{Re}\left(f^{\prime}(q)^{-1} q f^{\prime \prime}(q)\right)>-1, \quad \forall q \in \mathbb{B},
$$

then, by Proposition $3.6 g$ is slice regular on $\mathbb{B}$ with $\operatorname{Re} g(q)>-1$ for all $q \in \mathbb{B}$. Then

$$
\left|(2+g(q))^{-1} g(q)\right|<1, \quad \forall q \in \mathbb{B} .
$$

Consider the slice regular function

$$
h(q)=(2+g(q))^{-*} * g(q)=\left(2 f^{\prime}(q)+q f^{\prime \prime}(q)\right)^{-*} *\left(q f^{\prime \prime}(q)\right), \quad \forall q \in \mathbb{B},
$$

with $h(0)=h^{\prime}(0)=\ldots=h^{(m-1)}(0)=0$ and $|h(q)|<1$ on $\mathbb{B}$ by Proposition 3.6 again and (5.11). Whence the Schwarz lemma for slice regular functions in Theorem 3.10 implies that $|h(q)| \leq|q|^{m}$ for all $q \in \mathbb{B}$. Then

$$
\left|h \circ T_{2+g^{c}}(q)\right| \leq\left|T_{2+g^{c}}(q)\right|^{m}=|q|^{m}, \quad \forall q \in \mathbb{B} .
$$

That is, by Proposition 2.7

$$
\left|(2+g(q))^{-1} g(q)\right| \leq|q|^{m}, \quad \forall q \in \mathbb{B},
$$

equivalently,

$$
\left|g(q)-\frac{2 r^{2 m}}{1-r^{2 m}}\right| \leq \frac{2 r^{m}}{1-r^{2 m}}, \quad|q|=r<1,
$$

which implies that

$$
\left|g \circ T_{\left(f^{\prime}\right)^{c}}(q)-\frac{2 r^{2 m}}{1-r^{2 m}}\right| \leq \frac{2 r^{m}}{1-r^{2 m}}, \quad|q|=r<1,
$$

i.e., by Proposition 2.7

$$
\left|f^{\prime}(q)^{-1} q f^{\prime \prime}(q)-\frac{2 r^{2 m}}{1-r^{2 m}}\right| \leq \frac{2 r^{m}}{1-r^{2 m}}, \quad|q|=r<1 .
$$

Specially,

$$
\left|\operatorname{Re}\left(f^{\prime}(q)^{-1} q f^{\prime \prime}(q)\right)-\frac{2 r^{2 m}}{1-r^{2 m}}\right| \leq \frac{2 r^{m}}{1-r^{2 m}}, \quad|q|=r<1 .
$$

This together with (3.7) yields that

$$
-\frac{2 r^{m-1}}{1+r^{m}} \leq \frac{\partial}{\partial r} \log \left|f^{\prime}(r u)\right| \leq \frac{2 r^{m-1}}{1-r^{m}}, \quad q=r u .
$$

Integrating the above inequality from 0 to $r$ gives

$$
-\frac{2}{m} \log \left(1+r^{m}\right) \leq \log \left|f^{\prime}(r u)\right| \leq-\frac{2}{m} \log \left(1-r^{m}\right), \quad q=r u,
$$

as desired.

From Theorem 5.4 we deduce a precise growth theorem for $\mathcal{S}^{*}$.

Theorem 5.5. Let $f(q)=q+\sum_{n=m+1}^{+\infty} q^{n} a_{n} \in \mathcal{S}^{*}$ for some positive integer $m$. Then

$$
\frac{|q|}{\left(1+|q|^{m}\right)^{2 / m}} \leq|f(q)| \leq \frac{|q|}{\left(1-|q|^{m}\right)^{2 / m}}, \quad \forall q \in \mathbb{B} .
$$


In fact, estimates on the right side of (5.8) and (5.9) can be directly obtained from coefficient estimates in Theorem 1.1 Equality on the right side of (5.8) or (5.9) occurs for some $q_{0} \in \mathbb{B} \backslash\{0\}$, if and only if

$$
f(q)=q(1-q u)^{-* 2}, \quad q \in \mathbb{B}
$$

for some $u \in \partial \mathbb{B}$.

Moreover, those two estimates hold in a larger class. Let $f, g$ be two slice regular functions on $\mathbb{B}$. Write $f \prec_{\mathcal{N}} g$ if there exists $w \in \mathcal{N}(\mathbb{B})$ with $w(0)=0$ and $|w(q)|<1$ for all $q \in \mathbb{B}$ such that $f(q)=g(w(q))$ for all $q \in \mathbb{B}$ (see [16, Definition 2.10]). Note that the composition of slice regular functions, when defined, does not give generally a slice regular function. However, if $w \in \mathcal{N}(\mathbb{B})$, then the composition $f \circ w$ is slice regular for any slice regular function $f$. Since the proof of Theorem 5.6 below is similar to the classical case (see e.g., [13, p. 202]), we omit it here.

Theorem 5.6. Let $f, g$ be two slice regular functions on $\mathbb{B}$. If $f \in \mathcal{C}$ and $g \prec_{\mathcal{N}} f$, then the following inequalities hold for all $q \in \mathbb{B}$

$$
|g(q)| \leq \frac{|q|}{(1-|q|)^{2}} \text { and }\left|g^{\prime}(q)\right| \leq \frac{1+|q|}{(1-|q|)^{3}}
$$

In fact, we can obtain more information related to (5.8). Note that the author in 31 established the Hayman's regularity theorem making use of complex version of Theorem 5.7 for the $p$-univalent holomorphic function.

Theorem 5.7. Let $f \in \mathcal{S}^{*}$ and $M_{\infty}(r, f)=\max _{|q|=r}|f(q)|$ for $r \in(0,1)$. Then the function $\phi(r)=$ $\frac{1}{r}(1-r)^{2} M_{\infty}(r, f)$ is decreasing on $(0,1)$ and hence tends to a limit $\alpha \in[0,1]$. For any $\alpha \in[0,1]$, there exists $u \in \partial \mathbb{B}$ such that

$$
\lim _{r \nearrow 1^{-}}(1-r)^{2}|f(r u)|=\alpha .
$$

Proof. From the proof in Theorem 5.3, we have

$$
\frac{\left|f\left(r_{2} u\right)\right|}{\left|f\left(r_{1} u\right)\right|} \leq \frac{r_{2}\left(1-r_{1}\right)^{2}}{r_{1}\left(1-r_{2}\right)^{2}} .
$$

Choose $u \in \partial \mathbb{B}$ such that $M_{\infty}\left(r_{2}, f\right)=\left|f\left(r_{2} u\right)\right|$. Whence the above inequality yields that

$$
\frac{1}{r_{2}}\left(1-r_{2}\right)^{2} M_{\infty}\left(r_{2}, f\right) \leq \frac{1}{r_{1}}\left(1-r_{1}\right)^{2}\left|f\left(r_{1} u\right)\right| \leq \frac{1}{r_{1}}\left(1-r_{1}\right)^{2} M_{\infty}\left(r_{1}, f\right)
$$

that is to say $\phi(r)$ decreases on $(0,1)$ and hence tends to a limit $\alpha$. From (5.8), there holds that $\phi(r) \in[0,1]$, thus $\alpha \in[0,1]$.

Let $\left\{r_{n}\right\}$ be a sequence increasing to 1 and choose $u_{n} \in \partial \mathbb{B}$ such that $M_{\infty}\left(r_{n}, f\right)=\left|f\left(r_{n} u_{n}\right)\right|$. From the compactness of $\partial \mathbb{B}$, there exists a cluster point $u_{\infty} \in \partial \mathbb{B}$. From (5.12), we have, for $r<r_{n}$,

$$
\alpha \leq \frac{1}{r_{n}}\left(1-r_{n}\right)^{2}\left|f\left(r_{n} u_{n}\right)\right| \leq \frac{1}{r}(1-r)^{2}\left|f\left(r u_{n}\right)\right| \text {. }
$$

Letting $n \rightarrow \infty$, we obtain

$$
\alpha \leq \frac{1}{r}(1-r)^{2}\left|f\left(r u_{\infty}\right)\right| \leq \frac{1}{r}(1-r)^{2} M_{\infty}(r, f)
$$

as desired. 


\section{Radius Problems}

In this section, we consider some radius problems for slice regular functions.

6.1. Bohr Theorem. Based on Lemma 3.13 the authors in 12] established the quaternionic analog of the sharp version of the Bohr theorem saying that

Let $f(q)=\sum_{n=0}^{+\infty} q^{n} a_{n}$ be a slice regular function on $\mathbb{B}$, continuous on the closure $\mathbb{B}$, such that $|f(q)|<1$ for all $|q| \leq 1$. Then

$$
\sum_{n=0}^{+\infty}\left|q^{n} a_{n}\right|<1, \quad|q| \leq \frac{1}{3}
$$

Moreover $1 / 3$ is the largest radius for which the statement is true.

For various generalizations and variants of the Bohr theorem, see e.g., [1, 4, 10, 30] and references therein.

Inequality (6.1) also can be written as

$$
\sum_{n=1}^{+\infty}\left|q^{n} a_{n}\right|<\operatorname{dist}(f(0), \partial \mathbb{B}), \quad|q| \leq \frac{1}{3}
$$

From this view, we can give the Bohr theorem in a more general setting as follows.

Theorem 6.1. Let $f(q)=\sum_{n=0}^{+\infty} q^{n} a_{n}$ be a slice regular function on $\mathbb{B}$ such that $f(q) \in \Pi:=\{q \in \mathbb{H}$ : $\operatorname{Re} q \leq 1\}$ for all $q \in \mathbb{B}$. Then

$$
\sum_{n=1}^{+\infty}\left|q^{n} a_{n}\right| \leq \operatorname{dist}(f(0), \partial \Pi), \quad|q| \leq \frac{1}{3} .
$$

Proof. Under the condition of Theorem 6.1 it follows that (see [39, Theorem 4])

$$
\left|a_{n}\right| \leq 2(1-\operatorname{Re} f(0)), \quad n=1,2,3, \ldots,
$$

which implies that

$$
\sum_{n=1}^{+\infty}\left|q^{n} a_{n}\right| \leq 2(1-\operatorname{Re} f(0)) \sum_{n=1}^{+\infty}|q|^{n} \leq 1-\operatorname{Re} f(0)=\operatorname{dist}(f(0), \partial \Pi), \quad|q| \leq \frac{1}{3}
$$

as desired.

6.2. Rogosinski Theorem. The well-known theorem of Rogosinski asserts that if the modulus of the holomorphic function on $\mathbb{D}$ is less than 1 , then all partial sums of its power series are less than 1 on $\left\{z \in \mathbb{C}:|z|<\frac{1}{2}\right\}$ [44]. This theorem has been generalized to holomorphic mappings of the open unit ball with values in an arbitrary convex domain in [2] by using the following crucial step.

Lemma 6.2. Let $g_{m, r}(z)=\sum_{n=0}^{m} r^{n} z^{n}, z \in \mathbb{D}, r \in \mathbb{R}$. Then

$$
\operatorname{Re} g_{m, r}(z) \geq \frac{1}{2}
$$

for all $z \in \mathbb{D}$ and $r \in\left(0, r_{m}\right)$, where $r_{1}=1 / 2, r_{2}=\sqrt{3 / 8}$, and $r_{m}$ for $m \geq 3$ is the unique positive solution of the equation

$$
1-r-2 r^{m+1}=0 .
$$


Moreover,

$$
1-\frac{\log m}{m}<r_{m}<1-\frac{1}{m}, \quad m \geq 3
$$

Based on Lemma 6.2 we now establish the quaternionic analogy of the Rogosinski theorem as follows.

Theorem 6.3. Let $f=g+\bar{h}: \mathbb{B} \rightarrow \mathbb{H}$ be such that the image $f(\mathbb{B})$ is convex, where $g(q)=\sum_{n=0}^{+\infty} q^{n} a_{n}$ and $h(q)=\sum_{n=0}^{+\infty} q^{n} b_{n}$ are slice regular functions on $\mathbb{B}$. Then each partial sum

$$
S_{m}(q)=\sum_{n=0}^{m} q^{n} a_{n}+\overline{\sum_{n=0}^{m} q^{n} b_{n}}
$$

maps $B\left(0, r_{m}\right)$ into $f(\mathbb{B})$, where $r_{m}$ are given by Lemma 6.2.

Proof. For any $q \in \mathbb{B}_{I}$, it follows that

$$
S_{m}\left(r_{m} q\right)=\frac{1}{2 \pi} \int_{0}^{2 \pi}\left(1+2 \operatorname{Re} \sum_{n=1}^{m} r_{m}^{n} e^{n I \theta}\right) f\left(e^{I \theta} q\right) d \theta .
$$

Note that, for any fixed $I \in \mathbb{S}$ and each positive integer $m$, by Lemma 6.2

$$
\frac{1}{2 \pi}\left(1+2 \operatorname{Re} \sum_{n=1}^{m} r_{m}^{n} e^{n I \theta}\right) d \theta
$$

is a probability measure on the interval $[0,2 \pi]$ and the image $f(\mathbb{B})$ is convex, the desired result follows immediately.

6.3. One-Quarter Covering Theorem. As an application of (5.8) in Theorem 5.3, the Koebe type one-quarter covering theorem for the class $\mathcal{S}^{*}$ is obtained.

Theorem 6.4. Let $f \in \mathcal{S}^{*}$, then it holds that

$$
B\left(0, \frac{1}{4}\right) \subset f(\mathbb{B}) .
$$

The estimate is precise.

Proof. The first inequality in (5.8) implies that

$$
\liminf _{q \rightarrow \partial \mathbb{B}}|f(q)| \geq \frac{1}{4}
$$

From the open mapping theorem (see [22, Theorem 7.7]), the image set $f(\mathbb{B})$ is open containing the origin point 0 . This together with (6.3) and [48, Proposition 3.1] shows that $f(\mathbb{B})$ contains the ball $B(0,1 / 4)$

The preciseness of estimate can be shown by the slice regular function

$$
f(q)=q(1-q)^{-2}, \quad \forall q \in \mathbb{B} .
$$

Then

$$
\operatorname{Re}\left(f(q)^{-1} q f^{\prime}(q)\right)=\operatorname{Re}\left((1+q)(1-q)^{-1}\right)=\frac{1-|q|^{2}}{|1-q|^{2}}>0, \quad \forall q \in \mathbb{B},
$$

which shows that $f \in \mathcal{S}^{*}$. It is easy to show that $f(\mathbb{B})=\{q \in \mathbb{H}: q \notin(-\infty,-1 / 4]\}$ contains $B(0,1 / 4)$ while contains no ball centered at 0 with radius larger than $1 / 4$, as desired. 
Now we can offer another covering theorem from Theorem 5.5. instead of Theorem 5.3 . Specially, taking $m=2$ in Theorem 5.5 we have

Theorem 6.5. Let $f(q)=q+\sum_{n=3}^{+\infty} q^{n} a_{n} \in \mathcal{S}^{*}$, then it holds that

$$
B\left(0, \frac{1}{2}\right) \subset f(\mathbb{B}) .
$$

6.4. Bloch-Landau Theorem. In complex analysis, a well-known result of Bloch-Landau theorem says that if $F$ is a holomorphic function in $\mathbb{D}$ with the only restriction $F^{\prime}(0)=1$, then the image of $F$ contains a disc of radius $r \geq b$, where $b$ is an absolute constant.

Now we introduce its corresponding version of slice regular functions. For $a \in \mathbb{B}$ and the slice regular function $f$ on $\mathbb{B}$ with $f^{\prime}(0)=1$, denote by $r(a, f)$ the radius of the largest ball contained in the image of $f$ centered at $f(a)$ and denote $r(f)=\sup \{r(a, f), a \in \mathbb{B}\}$. In 48 , we have proved that $r(f)>0.23$ for any slice regular function $f$ with Bloch seminorm $\sup _{q \in \mathbb{B}}\left(1-|q|^{2}\right)\left|f^{\prime}(q)\right|=1$.

In this paper, we restrict functions $f$ in $r(f)$ to be slice regular functions with convex image. Define

$$
C=\inf \left\{r(f): f \text { is slice regular on } \mathbb{B} \text { such that } f^{\prime}(0)=1 \text { and } f(\mathbb{B}) \text { convex }\right\} \text {. }
$$

Let us prove Theorem 1.5 before giving the upper and lower bounds of $C$.

Proof of Theorem 1.5. Let $f$ be as described in the theorem and assume that $f(0)=0$ for otherwise we can consider the slice regular function $f-f(0)$. Let $p \in \partial f(\mathbb{B})$ be a point at minimum distance from the origin 0. From the open mapping theorem for slice regular function [17, we have $|p|>0$. If $|p|=+\infty$, the theorem has been proved. Otherwise, $0<|p|<+\infty$, we obtain that

$$
\operatorname{Re}(f(q) \bar{p})<|p|^{2}, \quad \forall q \in \mathbb{B},
$$

since $f(\mathbb{B})$ is convex.

Consider the slice regular function

$$
g(q)=\left(2|p|^{2}-f(q) \bar{p}\right)^{-*} * f(q) \bar{p}, \quad \forall q \in \mathbb{B} .
$$

Then it follows that $g(0)=0$ and $g^{\prime}(0)=(2 p)^{-1}$. From (6.5), we have

$$
|f(q) \bar{p}|<\left.|2| p\right|^{2}-f(q) \bar{p} \mid, \quad \forall q \in \mathbb{B},
$$

which implies, by 3.5 in Proposition 3.6 $|g(q)|<1$ for all $q \in \mathbb{B}$. The Schwarz lemma for slice regular functions in Theorem 3.10 implies that $\left|g^{\prime}(0)\right| \leq 1$. Hence, $|p| \geq 1 / 2$, which shows the image $f(\mathbb{B})$ contains the open ball $B(0,1 / 2)$. To see that the constant $1 / 2$ is optimal, we consider the slice regular function given by

$$
f(q)=q(1-q)^{-1}, \quad \forall q \in \mathbb{B} .
$$

It is easy to show that $f^{\prime}(0)=1$ and $f(\mathbb{B})=\{q \in \mathbb{H}: \operatorname{Re} q>-1 / 2\}$ is convex and contains the open ball centered at 0 of radius $1 / 2$ while $1 / 2$ is optimal, as desired.

As a consequence of Theorem 1.5, we give a Bloch-Landau theorem for slice regular functions with convex image.

Theorem 6.6. The constant $C$ given in (6.4) has the following estimate

$$
\frac{1}{2} \leq C \leq \frac{\pi}{4}
$$


Proof. From Theorem 1.5 it suffices to show that $C \leq \pi / 4$. To this end, let us consider the slice regular function

$$
f(q)=\sum_{n=0}^{+\infty} \frac{q^{2 n+1}}{2 n+1}, \quad q \in \mathbb{B}
$$

with $f^{\prime}(0)=1$.

Note that the image $f(\mathbb{B})=\left\{q \in \mathbb{H}:|\operatorname{Im} q|<\frac{\pi}{4}\right\}$ is convex, the desired result follows.

We have proved the Bieberbach conjecture over quaternions for slice close-to-convex functions. It is still an open problem if the Bieberbach conjecture holds true for injective slice regular functions.

Open question : Does it hold that $\left|a_{n}\right| \leq n, \quad n=2,3, \ldots$, provided that $f(q)=q+\Sigma_{n=2}^{+\infty} q^{n} a_{n}$ is an injective slice regular function in the open unit ball $\mathbb{B}$ ?

\section{References}

1. Y. Abu-Muhanna, R. M. Ali, S. Ponnusamy, On the Bohr inequality,Progress in approximation theory and applicable complex analysis, 269-300, Springer Optim. Appl., 117, Springer, Cham, 2017.

2. L. Aizenberg, M. Elin, D. Shoikhet, On the Rogosinski radius for holomorphic mappings and some of its applications, Studia Math. 168 (2005), no. 2, 147-158.

3. N. Arcozzi, G. Sarfatti, Invariant metrics for the quaternionic Hardy space, J. Geom. Anal. 25 (2015), no. 3, 2028-2059.

4. F. Bayart, D. Pellegrino, J. B. Seoane-Sepúlveda, The Bohr radius of the n-dimensional polydisk is equivalent to $\sqrt{(\log n) / n}$, Adv. Math. 264 (2014), 726-746.

5. C. Bisi, C. Stoppato, The Schwarz-Pick lemma for slice regular functions, Indiana Univ. Math. J. 61 (2012), 297-317.

6. H. Cartan, Sur la possibilité d'étendre aux fonctions de plusieurs variables complexes la théorie des fonctions univalents, in: P. Montel (Ed.), Leçons sur les Fonctions Univalents ou Mutivalents, Gauthier-Villars, Paris, 1933, pp. $129-155$.

7. F. Colombo, I. Sabadini, D. C. Struppa, Slice monogenic functions, Israel J. Math. 171 (2009), $385-403$.

8. F. Colombo, I. Sabadini, D. C. Struppa, Noncommutative functional calculus. Theory and applications of slice hyperholomorphic functions, Progress in Mathematics, vol. 289. Birkhäuser/Springer, Basel, 2011.

9. L. de Branges, A proof of the Bieberbach conjecture, Acta Mathematica, 154 (1985), 137-152.

10. A. Defant, M. Maestre, U. Schwarting, Bohr radii of vector valued holomorphic functions, Adv. Math. 231 (2012), no. 5, 2837-2857.

11. C. Della Rocchetta, G. Gentili, G. Sarfatti, A Bloch-Landau theorem for slice regular functions, Advances in hypercomplex analysis, 55-74, Springer INdAM Ser., 1, Springer, Milan, 2013.

12. C. Della Rocchetta, G. Gentili, G. Sarfatti, The Bohr theorem for slice regular functions, Math. Nachr. 285 (2012), no. 17-18, 2093-2105.

13. P. L. Duren, Univalent functions, 259. Springer-Verlag, New York, 1983.

14. M. Fekete, G. Szegö, Eine Bemerkung über Ungerade Schlichte Funktionen, J. London Math. Soc. 8 (1933), 85-89.

15. S. G. Gal, J. O. González-Cervantes, I. Sabadini, Univalence results for slice regular functions of a quaternion variable, Complex Var. Elliptic Equ. 60 (2015), no. 10, 1346-1365.

16. S. G. Gal, J. O. González-Cervantes, I. Sabadini, On some geometric properties of slice regular functions of a quaternion variable, Complex Var. Elliptic Equ. 60 (2015), no. 10, 1431-1455.

17. G. Gentili, C. Stoppato, The zero sets of slice regular functions and the open mapping theorem, Hypercomplex analysis and applications, 95-107, Trends Math., Birkhäuser/Springer Basel AG, Basel, 2011.

18. G. Gentili, D. C. Struppa, A new approach to Cullen-regular functions of a quaternionic variable, C. R. Math. Acad. Sci. Paris. 342 (2006) no. 10, 741-744.

19. G. Gentili, D. C. Struppa, A new theory of regular functions of a quaternionic variable, Adv. Math. 216 (2007) no. 1, 279-301.

20. G. Gentili, D. C. Struppa, Regular functions on the space of Cayley numbers, Rocky Mt. J. Math. 40 (2010), $225-241$.

21. G. Gentili, G. Sarfatti, Landau-Toeplitz theorems for slice regular functions over quaternions, Pacific J. Math. 265 (2013), no. 2, 381-404. 
22. G. Gentili, C. Stoppato, D. C. Struppa, Regular functions of a quaternionic variable, Springer Monographs in Mathematics, Springer, Berlin-Heidelberg, 2013.

23. R. Ghiloni, A. Perotti, Slice regular functions on real alternative algebras, Adv. Math. 226 (2011), no. 2, 16621691.

24. S. Gong, The Bieberbach conjecture, AMS/IP Studies in Advanced Mathematics, 12. American Mathematical Society, Providence, RI, 1999.

25. S. Gong, Convex and starlike mappings in several complex variables, Mathematics and its Applications, 435. Kluwer Academic Publishers, Dordrecht; Science Press, Beijing, 1998.

26. A. W. Goodman, Univalent functions and nonanalytic curves, Proc. Amer. Math. Soc. 8 (1957), 598-601.

27. A. Gori, F. Vlacci, Starlikeness for functions of a hypercomplex variable, Proc. Amer. Math. Soc. 145 (2017), no. $2,791-804$.

28. I. Graham, G. Kohr, Geometric function theory in one and higher dimensions, Monographs and Textbooks in Pure and Applied Mathematics, 255. Marcel Dekker, Inc., New York, 2003.

29. I. Graham, D. Varolin, Bloch constants in one and several variables, Pacific J. Math. 174 (1996), no. 2, $347-357$.

30. H. Hamada, T. Honda, G. Kohr, Gabriela, Bohr's theorem for holomorphic mappings with values in homogeneous balls, Israel J. Math. 173 (2009), 177-187.

31. W. K. Hayman, The asymptotic behaviour of p-valent functions, Proc. London Math. Soc. (3) 5 (1955), $257-284$.

32. W. Kaplan, Close-to-convex schlicht functions, Michigan Math. J. 1 (1952), 169-185.

33. W. Koepf, On the Fekete-Szegö problem for close-to-convex functions, Proc. Amer. Math. Soc. 101 (1987), no. 1, 89-95.

34. P. K. Kythe, Complex analysis. Conformal inequalities and the Bieberbach conjecture, Monographs and Research Notes in Mathematics. CRC Press, Boca Raton, FL, 2016.

35. F. R. Keogh, E. P. Merkes, A coefficient inequality for certain classes of analytic functions, Proc. Amer. Math. Soc. 20 (1969), 8-12.

36. X. Liu, T. Liu, Q. Xu, A proof of a weak version of the Bieberbach conjecture in several complex variables, Sci. China Math. 58 (2015), no. 12, 2531-2540.

37. T. Liu, G. Ren, Growth theorem of convex mappings on bounded convex circular domains, Sci. China Ser. A 41 (1998), 123-130

38. M. O. Reade, On close-to-convex univalent functions, Michigan Math. J. 3 (1955). 59-62.

39. G. Ren, X. Wang, Carathéodory theorems for slice regular functions, Complex Anal. Oper. Theory 9 (2015), no. $5,1229-1243$.

40. G. Ren, X. Wang, Julia theory for slice regular functions, Trans. Amer. Math. Soc. 369 (2017), no. 2 , $861-885$.

41. G. Ren, X. Wang, The growth and distortion theorems for slice monogenic functions, Pacific J. Math. 290 (2017), no. 1, 169-198.

42. G. Ren, X. Wang, Z. Xu, Slice regular functions on regular quadratic cones of real alternative algebras, Trends in Mathematics, Birkhäuser, (2016), 227-245.

43. M. S. Robertson, On the theory of univalent functions, Ann. of Math. (2) 37 (1936), no. 2, 374-408.

44. W. Rogosinski, Über Bildschranken bei Potenzreihen und ihren Abschnitten, Math. Z. 17 (1923), $260-276$.

45. K. A. Roper, T. J. Suffridge, Convexity properties of holomorphic mappings in $\mathbf{C}^{n}$, Trans. Amer. Math. Soc. 351 (1999), no. 5, 1803-1833

46. C. Stoppato, Regular Möbius transformations of the space of quaternions, Ann. Global Anal. Geom. 39 (2011), no. 4, 387-401.

47. C. Stoppato, A new series expansion for slice regular functions, Adv. Math. 231 (2012), no. 3-4, $1401-1416$.

48. Z. Xu, X. Wang, On two Bloch type theorems for quaternionic slice regular functions, arXiv:1601.02338, 2016.

Zhenghua Xu, School of Mathematics, HeFei University of Technology, Hefei 230601, China

E-mail address: zhxu@hfut.edu.cn

Guangbin Ren, School of Mathematical Sciences, University of Science and Technology of China, Hefei 230026, CHINA

E-mail address: rengb@ustc.edu.cn 